\title{
AN ARITHMETIC FUNCTION
}

\section{BY LEONARD CARLITZ}

\section{Introduction. The function*}

$$
\psi(k, m)=\sum_{s t=k} \mu(s) m^{t},
$$

where $\mu(s)$ is the Möbius function, has the property

$$
\psi(k, m) \equiv 0(\bmod k),
$$

for arbitrary integral $m$. Gegenbauer $\dagger$ has generalized this by replacing $\mu(s)$ by an arbitrary integral-valued function $w(s)$ for which

$$
\sum_{s \nmid k} w(s) \equiv 0(\bmod k),
$$

for all $k$. Clearly (3) holds for the function $\mu(s)$. Since (1) is equivalent to

$$
\sum_{s \mid k} \psi(s, m)=m^{k}
$$

we put

(4) $W(k, m)=\sum_{s t=k} w(s) m^{t}=\sum_{s d e=k} w(s) \psi(e, m)=\sum_{t e=k} \psi(e, m) \sum_{s \mid t} w(s)$

and therefore by (2) and (3),

$$
W(k, m) \equiv 0(\bmod k),
$$

for all $\mathrm{m}$. Conversely it is easy to show, by an induction on $k$, that (5) implies (3). Indeed, if (3) holds for all integers $<k$, it follows from (4) and (5) that

$$
\psi(1, m) \sum_{s \mid k} w(s)=m \sum_{s \mid k} w(s) \equiv 0(\bmod k) .
$$

Since this must hold for all $m$, we may select an $m$ prime to $k$, and therefore we have (3).

* For references see Dickson's History of the Theory of Numbers, vol. 1, pp. 84-86. Cited as Dickson.

† See Dickson, p. 86. 
2. The Generalized Function. In the right member of (1) replace $m^{t}$ by an arbitrary integral-valued function $g(t)$, and define

$$
\psi(k)=\psi(k, g)=\sum_{s t=k} \mu(s) g(t) .
$$

From the definition it follows at once that

$$
g(k)=\sum_{s \mid k} \psi(s)
$$

and for $(h, k)=1$,

$$
\psi(h k)=\sum_{s t=k} \mu(s) \psi(h t)
$$

We shall now show that

$$
\psi(k) \equiv 0(\bmod k)
$$

for all integers $k$ if and only if

$$
g\left(p^{e} t\right) \equiv g\left(p^{e-1} t\right)\left(\bmod p^{e}\right)
$$

for all primes $p$ and all integers $t$. Clearly we may assume in (8) that $p \nmid t$. Then if we write $k=p^{e} K$, where $p \nmid K$, it is easily seen that (6) implies

$$
\psi\left(p^{e} k\right)=\sum_{s t=k} \mu(s)\left\{g\left(p^{e} t\right)-g\left(p^{e-1} t\right)\right\} .
$$

This shows that if $(8)$ holds, then $\psi(k) \equiv 0\left(\bmod p^{e}\right)$ for every $p^{e}$ that divides $k$. Hence (8) is certainly a sufficient condition. Interchanging $K$ and $t$ in (9), and then inverting, we get

$$
g\left(p^{e} K\right)-g\left(p^{e-1} k\right)=\sum_{s \mid k} \psi\left(p^{e} s\right)
$$

from which it follows that (8) is also a necessary condition that (7) hold. Note that if (8) holds for each of two functions, it holds also for their product.

If now we replace the $\mu(s)$ of (6) by an integral-valued function $w(s)$ for which (3) is satisfied, we may define

$$
W(k)=W(k, g)=\sum_{s t=k} w(s) g(t)
$$

as generalizing $\psi(k, g)$. Then as above, 


$$
W(k, g)=\sum_{s t=k} \psi(s, g) \sum_{d \mid t} w(d),
$$

and therefore if (7) and (3) hold, it follows that

$$
W(k, g) \equiv 0(\bmod k) .
$$

Conversely it can be proved that (10) and (7) imply (3); similarly (10) and (3) imply (7).

3. Connection with Irreducible Polynomials. As is well known, if in (1) we put $m=p^{n}$, the power of a prime, the resulting function $\psi\left(k, p^{n}\right)$ is $k$ times the number of irreducible polynomials of degree $k$ in a single indeterminate, and with coefficients in the Galois field $G F\left(p^{n}\right)$. More generally, the number of irreducible factorable polynomials* in $G F\left(p^{n}\right)$,

$$
G \equiv \prod_{j=1}^{k}\left(\alpha_{j 0}+\alpha_{j 1} x_{1}+\cdots+\alpha_{j s} x_{s}\right), \quad \prod_{j=1}^{k} \alpha_{j s} \neq 0,
$$

is $\psi\left(k, p^{n s}\right) / k$.

In the case of the general function $\psi(k)=\psi(k, g)$, for which (7) is assumed to hold, we consider a set of polynomials $M$ with coefficients in a field (the precise nature of which need not be defined). The degree of $M$ is assumed defined; the number of polynomials $M$ of fixed degree $m$ will be denoted by $f(m)$, $f(0)=1$. It is assumed that $M$ can be factored into a product of powers of irreducible polynomials (of the set) in essentially one way. If $\psi(k) / k$ be the number of irreducible polynomials $P$ of degree $k$, we shall show that

$$
m f(m)=\sum_{s=1}^{m} \psi(s)\{f(m-s)+f(m-2 s)+\cdots\},
$$

or what is the same thing,

$$
m f(m)=\sum_{s=1}^{m} g(s) f(m-s) .
$$

We put

$$
F(m)=\prod_{\operatorname{deg} M=m} M, \quad \Theta(m)=\prod_{\operatorname{deg} P=m} P,
$$

* Duke Mathematical Journal, vol. 2 (1936), pp. 660-670. 
so that $F(m)$ is the product of all the polynomials of degree $m$, $\Theta(m)$ the product of the irreducible polynomials. To express $F(m)$ in terms of $\Theta$, let

$$
M=P^{e} A, \quad P \nmid A,
$$

where $P$ is of degree $s$, say. Then by (13),

$$
F(m)=\prod_{P, e} P^{e \phi_{m}-e s(P)},
$$

the product extending over all $P$, $e$ such that $e s \leqq m$, and $\phi_{k}(P)$ denotes the number of polynomials of degree $k$, not divisible by $P$. Evidently

$$
\phi_{k}(P)=\left\{\begin{array}{l}
f(k) \text { for } k<s, \\
f(k)-f(k-s) \text { for } k \geqq s .
\end{array}\right.
$$

Thus (14) becomes

$$
F(m)=\prod_{P} P^{\Sigma_{e} e^{\phi_{m}-e s(P)}} ;
$$

the exponent in the right member is

$$
\begin{aligned}
& \{f(m-s)-f(m-2 s)\}+2\{f(m-2 s)-f(m-3 s)\}+\cdots \\
& \quad+r f(m-r s)=f(m-s)+f(m-2 s)+\cdots+f(m-r s),
\end{aligned}
$$

where $r=[m / s]$, the greatest integer $\leqq m / s$. Grouping together all $P$ of equal degree, we have finally

$$
F(m)=\prod_{s=1}^{m}\{\Theta(s)\}^{f(m-s)+\cdots+f(m-r s)} .
$$

Comparison of the degree of the two members of (15) leads to

$$
\begin{aligned}
m f(m) & =\sum_{s=1}^{m} \psi(s) \sum_{e=1}^{r} f(m-e s) \\
& =\sum_{e s \leqq m} \psi(s) f(m-e s) \\
& =\sum_{k \leqq m} f(m-k) \sum_{e s=k} \psi(s) \\
& =\sum_{k=1}^{m} f(m-k) g(k),
\end{aligned}
$$

so that we have proved both (11) and (12). 
4. The L.C.M. Property. In the paper previously referred to, the following formula appears incidentally:

$$
\sum_{[s, t]=k} \psi\left(s, p^{n}\right) \psi\left(t, p^{n}\right)=\psi\left(k, p^{2 n}\right),
$$

the summation on the left extending over all $s$, $t$ with least common multiple equal to $k$. This formula may be proved very easily; indeed it follows at once from a formula due to von Sterneck.*

Let $g_{1}(m), g_{2}(m)$ denote arbitrary arithmetic functions, and $g(m)=g_{1}(m) g_{2}(m)$. Then for $\psi(k, g)$ as defined by (6), von Sterneck's formula is

$$
\sum_{[s, t]=k} \psi\left(s, g_{1}\right) \psi\left(t, g_{2}\right)=\psi(k, g) .
$$

To prove this, consider the equivalent formula

$$
\sum_{k \mid m} \sum_{[s, t]=k} \psi\left(s, g_{1}\right) \psi\left(t, g_{2}\right)=\sum_{k \mid m} \psi(k, g) .
$$

The summation conditions on the left are equivalent to $s \mid m$, $t \mid m$, that is, $s$ and $t$ independently ranging over the divisors of $m$. Thus we have

$$
\sum_{s \mid m} \psi\left(s, g_{1}\right) \sum_{t \mid m} \psi\left(t, g_{2}\right)=g_{1}(m) g_{2}(m)=\sum_{k \mid m} \psi\left(k, g_{1} g_{2}\right),
$$

which proves (18), and therefore (17).

If in (17) we take

$$
g_{1}(s)=m^{s}, \quad g_{2}(s)=n^{s},
$$

the formula becomes

$$
\sum_{[s, t]=k} \psi(s, m) \psi(t, n)=\psi(k, m n)
$$

a direct generalization of (16).

Formula (17) may be generalized to the case of $m$ functions $g_{1}, \cdots, g_{m}, g=g_{1} g_{2} \cdots g_{m}$,

$$
\sum_{\left[s_{1}, \cdots, s_{m}\right]=k} \psi\left(s_{1}, g_{1}\right) \cdots \psi\left(s_{m}, g_{m}\right)=\psi(k, g),
$$

* See Dickson, p. 151. For details of the L.C.M. calculus, see D. H. Lehmer, American Journal of Mathematics, vol. 53 (1931), pp. 843-854. 
the summation extending over all sets $s_{1}, \cdots, s_{m}$, with least common multiple equal to 1 .

5. A Polynomial Analog of $\psi(k)$. It is easy to define analogs of $\psi(k)$ having the property (2). For example, for an algebraic field, we have*

$$
\psi(\mathfrak{m}, \beta)=\sum_{\mathfrak{a} \mathfrak{b}=\mathfrak{m}} \mu(\mathfrak{a}) \beta^{n(\mathfrak{b})} \equiv 0(\bmod \mathfrak{m}),
$$

where $\mathfrak{m}$ is an ideal and $\beta$ an integer in the field.

We now define an analog in the domain of polynomials in a single indeterminate, with coefficients in a $G F\left(p^{n}\right)$ :

$$
\psi(M, G)=\sum_{A B=M} \mu(A) G^{|B|} .
$$

Here $\mu(A)$ is the Möbius function for the polynomial domain, and the absolute value $|B|$ is defined by

$$
|B|=p^{n b}, \quad b=\operatorname{deg} B .
$$

Then it is easy to show that

$$
\psi(M, G) \equiv 0(\bmod M),
$$

for arbitrary polynomials $G$. For $M$ irreducible, (20) reduces to Fermat's theorem.

More generally if $g(M)$ is a function of the polynomial $M$ whose values are polynomials in $G F\left(p^{n}\right)$, we may define

$$
\psi(M, g)=\sum_{A B=M} \mu(A) g(B),
$$

and prove, exactly as above, that $\psi(M, g) \equiv 0(\bmod M)$ if and only if

$$
g\left(P^{e} M\right) \equiv g\left(P^{e-1} M\right)\left(\bmod P^{e}\right) .
$$

Generally speaking, all our results for $\psi(m, g)$ carry over to the polynomial $\psi(M, g)$. In particular this is true of the L.C.M. property. The one exception is $\$ 3$; there seems to be no connection between $\psi(M, G)$ and classes of irreducible polynomials.

Duke UnIVERsity

\footnotetext{
* Due to J. Westlund; see Dickson, p. 86.
} 